\title{
THE ANTHROPOLOGY OF PUBLIC HEALTH
}

\author{
MELISSA PARKER* AND IAN HARPER $†$ \\ *International Medical Anthropology Programme, Brunel University and $\uparrow$ School of \\ Social and Political Studies, Edinburgh University
}

\section{Introduction: why have a special issue devoted to the anthropology of public health?}

The Journal of Biosocial Science regularly publishes papers addressing the social and cultural aspects of disease, sickness and well-being. Most of these papers attempt to understand the prevalence and distribution of disease and sickness within and between populations as well as local responses to biomedical interventions and public health policy more generally. They fall broadly within the remit of human ecology; and they embrace a 'factorial' model of disease in which social and cultural factors are deemed to be just one of a number of factors to be considered alongside a range of other factors. These include biological features of the infecting organism; nutritional factors; environmental factors; psychological factors; and genetic factors influencing susceptibility to disease at an individual and population level.

The authors contributing to this special issue share a sense of disquiet at the continuing predominance of this approach. They recognize that it is vital to document and understand the changing distribution of disease(s) within and between populations as, apart from anything else, effective public health policy cannot be formulated without such information. They also recognize that no single discipline has a monopoly of insight; and that effective public policy cannot be formulated without harnessing insights from the biological and social sciences.

However, working with a 'factorial' model of disease is problematic as it embraces conceptual splits that may be more misleading than they are helpful. To start with, a separation is implicitly made between 'science' and 'culture'. Leaving aside the question of what these two complex terms might mean, the suggestion is made that scientific knowledge and endeavour is quite separate from the context in which it is used or the social circumstances to which it might be applied. There is little, if any, recognition of the fact that what appears, in any specific context, to be 'scientific knowledge' has emerged and been shaped by multiple and overlapping ways of thinking and seeing embedded in complex social processes. These processes include power relations and economic hierarchies as well as conceptions generated by previous public health interventions.

The mainstream public health literature also makes a conceptual split between 'science' and 'culture'. Analysis is dominated by the combination of the 'factorial' model with biomedical epistemology and conventions of clinical practice. This type of approach typically reduces the investigation of social and cultural aspects of disease 
to discrete, static, quantifiable 'beliefs' held by (or sometimes even about) the study population. The separation of these beliefs from the ideas of medical science and its intended humanistic outcomes has led researchers to investigate 'barriers' to the effective provision of health care services. Surveys documenting the 'knowledge, attitudes and practices' of the study population and, more recently, 'focused ethnographic studies' have sought to understand the gaps between the provision and uptake of services in the health sector; and emphasis is frequently given to 'cultural factors' inhibiting their effective delivery.

There is, too, a persistent under-estimation of the ways in which public health discourses and biomedical ways of seeing (which are generally linked to the entrenched hierarchies of clinical practice) construct rather than discover 'knowledge', and are embedded in the social circumstances they observe. As Farmer (1999) has shown, the adoption of culture as a kind of variable in public health discussion has actually detracted from the development of broader understandings of the ways in which political, economic and social processes influence the prevalence, distribution and response to disease(s) within and between populations. Indeed, Farmer and many others (including Ogden, 1999; Baer et al., 2003) have shown that a critical medical anthropology can offer much more than an understanding of social and cultural factors. It can, and should, 're-configure the boundaries of the problem', offering (new) conceptual frameworks, substantive knowledge and methodological insights (Lambert \& McKevitt, 2002).

There are implications here for our own discipline of anthropology, and specifically medical anthropology. A great deal of funding allocated for research in medical anthropology comes from organizations committed to the 'factorial' model; and many colleagues have been drawn into work that fits this scheme (see, for example, Gove \& Pelto, 1994; and many of the papers in the book edited by Hahn, 1999). Their research can usefully be described as 'anthropology in public health'. It is our view that anthropological research that sets out to generate data that fits within pre-existing categories embraced by the 'factorial' model frequently compromises what anthropology has to offer as a discipline.

We feel that there is a need for an 'anthropology of public health' which remains passionately concerned about ill-health and deprivation and the need for public policy; but also remains committed to a rigorous and critical analytical perspective. In other words, an 'anthropology of public health' should not be divorced from the world of practitioners. It should engage and comment upon public policy in such a way that it helps to alleviate the distress and suffering caused by infection and disease. It should also comment on the unintended effects of such policy for the supposed beneficiaries, and the beliefs and practices of the health professionals themselves.

All the contributors to this special issue are committed to developing an 'anthropology of public health'. Their work draws upon a long tradition of research within medical anthropology, dating back to Paul's key text Health, Culture and Community (Paul, 1955) and the classic work of social anthropologists such as Evans Pritchard (1937). There is insufficient space to review the texts that inform our writing, but readers wishing to acquaint themselves with this literature will find the following articles and books helpful: Jansen (1978); Comaroff (1985); Dunn \& Janes (1986); Farmer (1992, 2003); Kane \& Mason (1992); Scheper-Hughes (1992); Good 
(1994); Parker (1995); Young (1995); Brandt (1997); Inhorn \& Brown (1997); Kleinman et al. (1998); Nichter \& Nichter (1996); Ellison et al. (2003).

We are all committed to the public health project. However, it is often an uphill struggle to make an effective contribution to public policy - not least because biomedical research, undertaken within a positivist framework, is typically given far more weight than the more interpretive social sciences (Kleinmann, 1995). By interacting with more positivist research and disciplinary practices, we are invariably called upon to defend anthropological methods; we finish this introduction with some comments on this.

Our articles draw upon a wide range of methods: open-ended, unstructured interviews; semi-structured interviews; surveys; historical and contemporary sources such as newspaper articles, public policy documents and so on. With the exception of the paper by Outram and Ellison, they are all informed by extensive periods of participant observation fieldwork. A great strength of drawing upon multiple methods whilst undertaking participant observation fieldwork is that it frees researchers to undertake research in an open-ended way; to define and re-define the questions as they grapple with trying to understand the interaction between people and ideas. Ample space can thus be given to eliciting and understanding local beliefs, behaviours and debates, whilst ensuring that they are not treated as discrete and divorced from the policies, actions and interventions of outsiders.

It is not possible to undertake this type of research without spending time talking to people in the field, participating in their daily activities, finding out about the many issues that concern them; and developing as detailed an understanding as possible of the context in which disease and illness is experienced. New and important questions (that could not have been envisaged from secondary sources) typically emerge by working in this way. It is for this reason that so many medical anthropologists remain sceptical of so much of the information published by social scientists working in more structured and quantitative ways. To their minds, and ours, heavy reliance upon pre-designed questions, combined with spending limited periods of time in the field, inevitably structures the 'qualitative' in terms defined by the researcher rather than the researched; and this may well be at the expense of understanding the very people they seek to assist. Moreover, the opportunities are not created to recognize and understand why it is that people will discuss some issues in one context but not another; and the type of issues that cannot be discussed (other than in the most superficial and misleading of ways) without first establishing rapport and trust with the researcher.

It is not unusual for many quantitatively orientated social scientists and social epidemiologists to respond to these concerns by saying that the ethnographic research undertaken by anthropologists is too subjective and anecdotal to be helpful. They see no reason why public policy should be informed by 'insights' emerging from ethnographic research when there is no way of knowing whether these insights are based upon a representative sample of the study population. Case studies, for example, may generate interesting information, but it is often unclear how much weight to give them as it is hard to gauge whether they capture the views of a select minority or represent the views of the majority.

To our minds, the dismissal of ethnographic research as subjective and anecdotal is erroneous. The generation of any 'fact', including 'objective' ones, is a process of 
complex interpretive strategies (Kleinman, 1995); and it is now widely held by medical anthropologists that all research, including that mediated by complex laboratory techniques, is subjective and interpretive. Apart from anything else, the types of things that a researcher sees and records (as well as the types of things that are left unrecorded or not seen at all) are, inevitably, influenced by the cultural and disciplinary background of the researcher and their research environment.

It is also important to note that the process of doing ethnographic research, including the selection of the field site, taking field notes, reflection upon these notes both in the field and thereafter, as well as drafting and re-drafting papers and ethnographic monographs is a complex and time-consuming task. The final product, particularly evident in the papers by Tim Allen and Suzette Heald in this special issue, is the end-point of a series of interpretive links informed by years of experience and reflection. To dismiss such work as subjective and anecdotal is to miss the point. It is the end-product of a continuous and iterative engagement with multiple ethnographic encounters leading to the interpretations on offer. This is also the case for those researching complex policy environments - notably, the world of state and international bureaucracies - where new forms of relationships and research strategies are required.

Finally, we end on a note of caution: we have observed in recent published literature an increasing tendency to categorize open-ended interviews as ethnographic. To be ethnographic, however, interviews should be conducted within the broader context of an ethnographic enquiry. The term should be reserved for this; and it is insufficient for researchers to suggest that they have moved beyond the factorial model of disease because they have undertaken some one-off, open-ended interviews and a handful of focus group discussions.

In sum, restricting the contribution of the social sciences, and anthropology in particular, to the study of 'social and cultural factors' is far too limiting and, in many cases, generates misleading and inaccurate data. The articles published in this special edition of the Journal of Biosocial Science have been selected to demonstrate some of the ways in which medical anthropologists, developing an 'anthropology of public health', can usefully contribute to the formulation of effective public health policy and practice. We very much hope that it will inspire future contributors to the journal to follow our lead!

\section{References}

Baer, H. A., Singer, M. \& Susser, I. (2003) Medical Anthropology and the World System: A Critical Perspective. Praeger, Westport.

Brandt, A. (1987) No Magic Bullet: A Social History of Venereal Disease in the United States Since 1880. Oxford University Press, New York.

Comaroff, C. (1985) Body of Power, Spirit of Resistance: The Culture and History of a South African People. University of Chicago Press, Chicago.

Dunn, E. \& Janes, C. (1986) Introduction: Medical Anthropology and Epidemiology. In Janes, C., Stall, R. \& Gifford, S. (eds) Anthropology and Epidemiology: Interdisciplinary Approaches to the Study of Health and Disease. Reidal, Dordrecht, pp. 1-34. 
Ellison, G., Parker, M. \& Campbell, C. (2003) Learning from HIV and AIDS. Biosocial Society Symposium Series. Cambridge University Press, Cambridge.

Evans Pritchard, E. E. (1937) Witchcraft, Oracles and Magic among the Azande. Clarendon Press, Oxford.

Farmer, P. (1992) AIDS and Accusation: Haiti and the Geography of Blame. University of California Press, Berkeley, Los Angeles and London.

Farmer, P. (1999) Infections and Inequalities: The Modern Plagues. University of California Press, Berkeley, Los Angeles and London.

Farmer, P. (2003) Pathologies of Power: Health, Human Rights, and the New War on the Poor. University of California Press, Berkeley, Los Angeles and London.

Good, B. (1994) Medicine, Rationality and Experience. Cambridge University Press, Cambridge.

Gove, S. \& Pelto, G. (1994) Focused ethnographic studies in the WHO programme for the control of acute respiratory infections. Medical Anthropology 15, 409-424.

Hahn, R. (ed.) (1999) Anthropology in Public Health: Bridging Differences in Culture and Society. Oxford University Press, New York.

Inhorn, M. \& Brown, P. (eds) (1997) The Anthropology of Infectious Disease: International Health Perspectives. Gordon and Breach Publishers, Amsterdam.

Jansen, J. M. (1978) The Quest for Therapy in Lower Zaire. University of California Press, Berkeley.

Kane, S. \& Mason, T. (1992) 'IV drug users' and 'sex partners': the limits of epidemiological categories and the ethnography of risk. In Herdt, G. \& Lindenbaum, S. (ed.) The Time of AIDS: Social Analysis, Theory and Method. Sage Publications, London, pp. 199-222.

Kleinman, A. (1995) Writing at the Margin. University of California Press, Berkeley, Los Angeles and London.

Kleinman, A., Das, V. \& Lock, M. (eds) (1998) Social Suffering. University of California Press, Berkeley.

Lambert, H. \& McKevitt, C. (2002) Anthropology in health research: from qualitative methods to multidisciplinarity. British Medical Journal 325, 210-213.

Nichter, M. \& Nichter, M. (1996) Anthropology and International Health: Asian Case Studies. Gordon and Breach Science Publishers, Amsterdam.

Ogden, J. (1999) Compliance versus adherence: just a matter of language? The politics and poetics of public health. In Porter, J. \& Grange, J. (eds) Tuberculosis: An Interdisciplinary Perspective. Imperial College Press, London.

Parker, M. (1995) Rethinking female circumcision. Africa 65(4), 506-523.

Paul, D. B. (1955) Health, Culture, and Community: Case Studies of Public Reactions to Health Programmes. Russell Sage Foundation, New York.

Scheper-Hughes, N. (1992) Death Without Weeping: The Violence of Everyday Life in Brazil. University of California Press, Berkeley, Los Angeles and London.

Young, A. (1995) The Harmony of Illusions: Inventing Post-traumatic Stress Disorder. Princeton University Press, Princeton, New Jersey. 